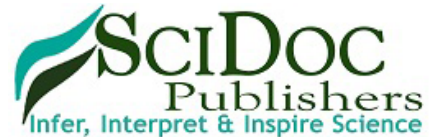

International Journal of Surgery and Research (IJSR)

ISSN: 2379-156X

\title{
Gender Differences in the Care of Cleft Lip and Palate Patients at Sohag Craniofacial Center
}

Research Article

Saied S*, Ahmed A Aggag, Bakri S

Sohag Faculty of Medicine, Plastic Surgery Department, Sohag University, Sohag, Egypt.

\section{Abstract}

Gender plays an important role in health care with direct and indirect effect in provided medical and surgical care; the aim of the study was to investigate if there is any difference in the surgical treatment of patients with cleft lip and palate between males and females in our community.

This is a retrospective study of 864 patients with variable ages, varieties and sex with total number of 1674 surgical procedures, Each procedure categorized as primary surgery (primary lip and palatal repair) or secondary surgery (aesthetic lip revision and palatal functional revision as palatal fistula or VPI ). Cases were categorized according to extent to UCLP, BCLP, UCL, BCL, CSP, CSHP. Total number of procedures for each diagnosis and the age of first surgical procedure is then analyzed and compared according to gender of patients, the result of our study show that females received more secondary surgeries than men. But this difference in secondary surgeries is statistically significant only in BCLP and UCLP cases, which underwent statistically significant more number of lip and nose revisions with no statistically significant difference in secondary palatal re repairs, also no statistically significant differences in the number of primary surgeries or the age of first operation. This study give an alert about the care given with more secondary lip and nose revisions done in females meaning that females indications of surgery may be over estimated or the males indications may be neglected, Many interpretations, explanations for this results and further studies also needed.

Abbreviations: CL: Cleft Lip; CP: Cleft Palate; CLP: Cleft Lip and Palate; SUH: Sohag University Hospital; CSP: Cleft Soft Palate; CSHP: Cleft Soft and Hard Palate; UCLP: Unilateral Cleft Lip and Palate; BCLP: Bilateral Cleft Lip and Palate; S.D: Standard Deviation; S.E.M: Standard Error Mean; Pr.: Procedure.

\section{Introduction}

Orofacial clefts including cleft lip (CL), cleft lip and palate (CLP), and cleft palate $(\mathrm{CP})$ alone are the most common cranio-maxillofacial birth defect. It can markedly affect the morphology and almost all the functions in the facial area, Although not a major cause of mortality, CLP does cause considerable morbidity to patient and causes a financial load for families with a concomitant societal burden [1].

Reported data on the frequency of orofacial clefts vary according to the investigator and the country. The overall incidence of cleft lip with or without cleft palate is approximately 1 in 750 to 1000 live births, establishing it as one of the most common congenital anomalies [2].
The infant born with a cleft presents the plastic surgeon with a uniquely difficult surgical challenge that requires both aesthetic sense and technical skill to restore form and function. The complexity of the cleft deformity and the influence of facial growth also provide the surgeon with a uniquely rewarding opportunity to care for the cleft patient from infancy to young adulthood [3].

Individuals with CLP may experience problems with feeding, speaking, hearing and social integration, Patients will undergo multiple rounds of surgical repair starting in the first year of life and may continue until 18 or 20 years old. Frequently, extensive dental and orthodontic treatment, speech and hearing therapy may be required as well as referral for psychotherapy and genetic counseling [1].

Sex and gender are two terms that are often interchanged in com-

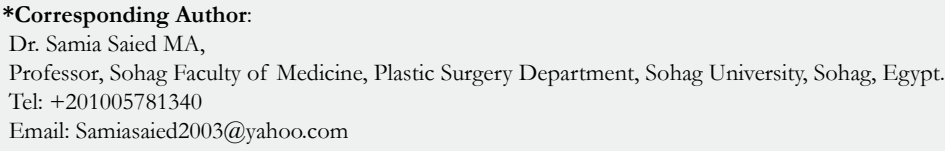

Received: November 27, 2019

Accepted: December 12, 2019

Published: December 16, 2019

Citation: Saied S, Ahmed A Aggag, Bakri S. Gender Differences in the Care of Cleft Lip and Palate Patients at Sohag Craniofacial Center. Int J Surg Res. 2019;6(3):136-140. doi: http://dx.doi.org/10.19070/2379-156X-1900029

Copyright: Samia Saied ${ }^{\circ}$ 2019. This is an open-access article distributed under the terms of the Creative Commons Attribution License, which permits unrestricted use, distribution and reproduction in any medium, provided the original author and source are credited. 
mon language without real knowledge about the complex differences between the two. Sex primarily refer to the biological aspect of being either a man or a woman, depending on several physical conditions, the most common of which is the presence of male or female genitalia [4].

Gender means socially constructed relationships, personality traits, attitudes, values, and influence that society ascribes to the two sexes on a differential basis. Gender characteristics do not exist in isolation, but are defined in relation to one another and through the relationships between women and men, girls and boys. Simply sex refers to biological differences, whereas gender refers to social differences [5].

Gender analysis in health has been undertaken mainly by scientists who noted that biological differences alone cannot adequately explain health behavior. Health outcomes also depend upon economic and social factors that, in turn, are influenced by political and cultural conditions in society. To understand health and illness, both sex and gender must be taken into account [6].

Because sex and gender affect a wide range of physiological functions, they may have an impact on care of patients. There are a significant number of sex and gender differences for many common diseases and disorders that need to be considered in health care, public health strategies, regulations and training of medical personnel. Unfortunately, they are largely neglected. Despite the wealth of data on differences, medical practice does not sufficiently take gender into account in diagnosis, treatment or management of patients [7].

In last decade a considerable amount of research has been conducted in the area of health and gender, including gender differences and its effect in specific health conditions. Gender has been shown to influence how health policies are conceived and performed and how the health system responds to male and female patients [8].

The gender differences in the biological determinants of health and illness include genetic susceptibility to illness, hormonal and reproductive factors, and differences in physiological characteristics during life-cycle increase questions about gender differences in heart disease, mental illness, and osteoporosis with important recommendation that women be included in clinical studies to uncover gender differences and their impact on the prevention, diagnosis, and treatment of disease. Such recommendations are still not fully implemented [9].

Several conclusions regarding the value of gender for understanding many health conditions can be derived from several studies. First, gender clearly plays a role in the determinants and consequences of health problems, and it can no longer assume that a male model for health also applies to female model. The way in which gender affects these determinants and consequences may vary according to the conditions selected and the characteristics of the population studied. However, gender analysis is key to understand many differences in health care [6].

Although much is known about adults and children with cleft malformations, more remains to be learned, life satisfaction as well as function during management of cleft cases have been studied, there are no general directives regarding the impact of gender in patients with clefts [10].

Studies to determine gender bias in cleft cases have been done one of those including 98 patient with unilateral cleft lip and palate, 36 women and 62 men in which there was a statistically significant difference between male and female patients in number of secondary surgical procedures where females had an increased number compared to males $(\mathrm{p}=0.018)$. The difference appeared in the aesthetical surgeries of lip and nose. Female patients got $55 \%$ more secondary lip procedures $(\mathrm{p}=0.028)$ and $72 \%$ more secondary nose procedures compared to male patients $(\mathrm{p}=0.015)$. There was no significant difference in the number of primary surgical procedures $(p=0.923)[11]$.

The aim of the present study was to investigate if there is any difference in the surgical treatment of patients with cleft lip and palate between males and females.

\section{Patient and Methods}

This is a retrospective study that was carried out on patients that had been managed in Cleft and Craniofacial Unit, Plastic surgery department, sohag university hospital for cleft lip, cleft palate or cleft lip and palate taking in consideration gender of cases. All cases of cleft lip, cleft palate or cleft lip and palate that presented to and had surgical treatment with different varieties, sex and ages. We investigated 1168 cleft patients (593 female and 575 male) of which 304 patients were excluded because of incomplete medical files, so the total number of included cases was 864 cases of which 431 female and 433 male, with total number of 1674 surgical procedures of which 848 surgical procedures in males and 826 surgical procedures in females.

\section{Collection of data}

Patients were evaluated by a Sohag multi-disciplinary cleft team that included plastic surgeons, pediatricians, dentists, otolaryngologists and orthodontists.

Data for each patient was then registered in a Filemaker Pro Advanced version 12, database (Filemaker, Inc., Santa Clara, CA). The program designed with sheets for registration and follow up of cleft cases with different pages for; Personal information, preoperative treatments, cleft extent, congenital anomalies. Also, Operative data and images with speech, orthodontics, and hearing assessments are included. The data was collected from medical records archive of the Cleft and Craniofacial Unit, Plastic surgery department, Sohag University Hospital.

All surgical procedures of each patient were registered. Primary and secondary procedures were registered as well as anatomical region of the surgical procedure; lip, nose or palate, in total of 1674 interventions. Ages of first surgical procedure calculated.

The total number of cases was divided according to cleft extent to get a comparable population. The data of unilateral cleft lip and palate and bilateral cleft lip and palate used to compare between males and females regarding surgical interventions.The registered data was transferred to Microsoft Excel 2007 where it further was processed. 


\section{Statistical methods}

Statistical calculations were performed using SPSS Software for Windows (version 22.0, SPSS Inc., Chicago, IL, United States). Descriptive statistics, along with a variety of statistical tests and student's T-test. The $\mathrm{P}$ value was two-tailed and significance set to $\leq 0.05$.

\section{Result}

As regard health care males receives more medical treatments than females because of social, economic and biological factors, also focusing on the male model rather than a female one to compare a medical or surgical methods of treatments increasing the gape of care between both [6], the result in my study found that females received more surgical care than males in UCLP and BCLP. But no significant difference in cases with isolated cleft lip or isolated cleft palate.

As the same protocol for surgical care in our unite was applied to all cases whether male or female without any differentiation or discrimination, the primary surgical care was nearly equal with statistically no significant difference in number of primary surgical occasions.

The age of first operation done for primary correction of the lip and palate deformity was analyzed for both males and females, first the mean of operations calculated then statistical comparison done between both means, showing that there was no significant difference in the age of the first surgical procedure between males and females as both came for surgical correction nearly at the same age in males, 22.2 and females, 26.76\}, with $\mathrm{P}$ value $=$ 0.172 , but the mean of the age of the first operation found to be higher than our protocol which is 3 months for lip repair and 9 months for palatal repair.

To explain this high mean of age of first operation close search in the age for primary repair done finding that some cases had postponed their first operation with range of ( 335 months for females and 431 months for males).

This late presentation of some cases resulting in higher mean than our designed protocol, This late presentation can be explained by economical, religious, educational factors for those cases. (Table 2)
The suggested causes for this late presentations include the expensive cost of operations, post operative care and treatments, also parents think that his child may not with stand operations at this young age and prefer to postpone any surgical correction till patient reach 2 years and some time school age or even more specially with minor deformities as incomplete lip or cleft soft palate, Also this late presentation found in males and females suggesting that it's not related to gender discrimination.

As there is no single protocol for secondary surgical procedures many factors can affect this decision including patients, parents and surgeons. A study of gender difference in cleft surgical care done in Sweden resulted in that There was no significant difference between males and females as regard number of primary surgical procedures but females had significantly more secondary surgical procedures than males do and this difference can be found in lip revisions and not in palatal rerepaires [11].

The result in our study on UCLP and BCLP found that Females had significantly more secondary lip and nose operations than men but no significant difference found in palatal secondary operations, this may indicate that care received for aesthetical regions was more than those of speech, this can be explained by assuming that primary surgical correction of female patients is less successful than of male patients, but at the same time there is no evidence to support this hypothesis. As the operative techniques show no discrimination between both sex whether in procedure itself nor in the post operative care and treatments received, this make our thinking is directed to social factors rather than a biological and technical difference.

A study of adult cleft lip and palate patients found that females expressed a desire for further treatment twice as often as men [12].

We can assume that females have Internal motivations to do cosmetic revisions include the desire to diminish unpleasant feelings of depression, shame or social anxiety, the wish to alter disliked scars and deformities make them asking for better appearance than males do.

A study done on cleft females patients reported that there is significantly more dissatisfaction with their facial appearance than another group without clefts [13], also females feel a higher pres-

Table 1. Statistical analysis of different categories.

\begin{tabular}{|c|c|c|c|c|c|c|c|c|c|c|c|c|c|c|c|c|c|c|}
\hline & \multicolumn{6}{|c|}{ Cleft lip and palate } & \multicolumn{6}{|c|}{ Isolated Cleft palate } & \multicolumn{6}{|c|}{ Isolated Cleft lip } \\
\hline & \multicolumn{3}{|c|}{ UCLP } & \multicolumn{3}{|c|}{ BCLP } & \multicolumn{3}{|c|}{ CSP } & \multicolumn{3}{|c|}{ CSHP } & \multicolumn{3}{|c|}{ UCL } & \multicolumn{3}{|c|}{ BCL } \\
\hline & $\begin{array}{c}\text { Male } \\
\text { mean } \\
\text { n.126 }\end{array}$ & $\begin{array}{c}\text { female } \\
\text { mean } \\
\text { n.82 }\end{array}$ & $\begin{array}{c}\mathbf{P} \\
\text { value }\end{array}$ & $\begin{array}{c}\text { Male } \\
\text { mean } \\
\text { n.93 }\end{array}$ & $\begin{array}{c}\text { female } \\
\text { mean } \\
\text { n.42 }\end{array}$ & $\begin{array}{c}\mathbf{P} \\
\text { value }\end{array}$ & $\begin{array}{c}\text { Male } \\
\text { mean } \\
\text { n.43 }\end{array}$ & $\begin{array}{c}\text { female } \\
\text { mean } \\
\text { n.69 }\end{array}$ & $\begin{array}{c}P \\
\text { value }\end{array}$ & $\begin{array}{c}\text { Male } \\
\text { mean } \\
\text { n.54 }\end{array}$ & $\begin{array}{c}\text { female } \\
\text { mean } \\
\mathrm{n} .124 \\
\end{array}$ & $\begin{array}{c}\mathbf{P} \\
\text { value }\end{array}$ & $\begin{array}{c}\text { Male } \\
\text { mean } \\
\text { n.91 }\end{array}$ & $\begin{array}{c}\text { female } \\
\text { mean } \\
\text { n.97 } \\
\end{array}$ & $\begin{array}{c}\mathbf{P} \\
\text { value }\end{array}$ & $\begin{array}{c}\text { Male } \\
\text { mean } \\
\text { n.20 }\end{array}$ & $\begin{array}{c}\text { female } \\
\text { mean } \\
\text { n.12 }\end{array}$ & $\begin{array}{c}P \\
\text { value }\end{array}$ \\
\hline Total op. & 2.5 & 2.92 & 0.29 & 2.6 & 3.2 & 0.01 & 1.04 & 1.16 & 0.12 & 1.56 & 1.74 & 0.25 & 1.2 & 1.3 & 0.141 & 1.5 & 1.5 & 1 \\
\hline Primary & 2.02 & 2.1 & 0.242 & 2.1 & 2.3 & 0.067 & 1 & 1 & 1 & 1.07 & 1.11 & 0.43 & 1.01 & 1.01 & 0.96 & 1.1 & 1 & 0.272 \\
\hline secondary & 0.5 & 0.8 & 0.021 & 0.55 & 0.88 & 0.042 & 0.047 & 0.16 & 120 & 0.48 & 0.63 & 0.28 & 0.22 & 0.33 & 0.12 & 0.4 & 0.5 & 0.665 \\
\hline Sec. palate & 0.44 & 0.62 & 0.123 & 0.39 & 0.45 & 0.603 & & & & & & & & & & & & \\
\hline \multirow[t]{2}{*}{ Sec. lip } & 0.06 & 0.18 & 0.014 & 0.16 & 0.43 & 0.004 & & & & & & & & & & & & \\
\hline & \multicolumn{6}{|c|}{$\begin{array}{c}\text { Females had significantly more secondary lip and } \\
\text { nose opearations than men in both UCLP and } \\
\text { BCLP } \\
\text { There was no significant difference between males } \\
\text { and females in primary and secondary palatal } \\
\text { operations }\end{array}$} & \multicolumn{6}{|c|}{$\begin{array}{c}\text { There was no statistically significant difference } \\
\text { between males and females in primary and } \\
\text { secondary palatal operations }\end{array}$} & \multicolumn{6}{|c|}{$\begin{array}{l}\text { There was no statistically significant difference } \\
\text { between males and females in primary and sec- } \\
\text { ondary palatal operations }\end{array}$} \\
\hline
\end{tabular}


Table 2. The age of the first surgical procedure.

\begin{tabular}{|c|c|c|c|c|c|c|c|c|c|c|}
\hline Age & $\begin{array}{c}\text { To 9 } \\
\text { m }\end{array}$ & $\begin{array}{c}\mathbf{9 . 5}-\mathbf{1 2} \\
\text { month }\end{array}$ & $\begin{array}{c}\mathbf{1 3 - 1 8} \\
\text { month }\end{array}$ & $\begin{array}{c}\mathbf{1 9 - 2 4} \\
\text { month }\end{array}$ & $\begin{array}{c}\text { to 3 } \\
\text { years }\end{array}$ & $\begin{array}{c}\text { to 5 } \\
\text { years }\end{array}$ & $\begin{array}{c}\text { to 10 } \\
\text { years }\end{array}$ & $\mathbf{1 1 - 1 5}$ & $\mathbf{1 6 - 2 0}$ & $\mathbf{2 1 - 3 6}$ \\
\hline $\begin{array}{c}\text { N. of } \\
\text { cases }\end{array}$ & 514 & 90 & 63 & 29 & 30 & 51 & 51 & 16 & 8 & 12 \\
\hline percent & $60 \%$ & $10.3 \%$ & $7.2 \%$ & $3.3 \%$ & $3.4 \%$ & $6 \%$ & $6 \%$ & $1.7 \%$ & $0.8 \%$ & $1.30 \%$ \\
\hline
\end{tabular}

sure to look good, and are more stressed about their appearance, So females undergo more secondary surgeries to look better so they are able to face the society with confidence.

Also external motivators as parents of a female child are seeking for more lip and nose revisions because of educational and cultural reasons as marriage, they think that facial scars may decrease her chances in marriage, making society think that their offspring with congenital and deformed defects, this affect the family negatively. Also, the facial scars in a female cleft child make her under psychological pressure of un acceptance from her classmates and neighbors, giving more motivation to seek for more secondary operations.

Doctors also play a great role in cleft patients care, they may tend to perform more secondary operations for females than in males by being more responsive to patients and parents expectations and may suggest aesthetical corrections to a female patient more than a male one, As doctors are part of the society they are affected by his motivations and culture with every case is considered as a unique challenge forcing doctors to do more surgical interventions specially for females.

At the other hand males may not be concerned about their appearance as females or they may be afraid of the society look for males having aesthetic operations just to look more beautiful from their point of view, also because of economical reasons males have more financial duties towards their families so they avoid to undergo secondary aesthetical expensive operations with the money needed for it and the time needed for postoperative rehabilitation before return to normal activities and work.

The statistically insignificant difference in secondary surgical care of cleft palate patients in both males and females can be explained with the hypothesis that both has the equal concern and motivation for doing palatal re repairs to improve their speech, As speech is important in communication with the society and work colleges, but still females mean for secondary palatal operations higher than males, as they do more operations but still statistically insignificant.

Also data collected for isolated cleft palate with deferent severities (CSP ,CSHP) show that there is no statistically significant difference between males and females in primary and secondary surgical care of cases, which support the hypothesis of equal concern about speech quality in males and females, which is the same result obtained statistically in secondary palatal repairs in cases with cleft lip and palate (UCLP, BCLP).

There is no statistically significant difference between males and females in surgical care of cases with isolated cleft lip (UCL or BCL) both nearly have equal care. But the mean for female with UCL (0.33) is still higher than male (0.22). Also mean for revi- sions in female patients with BCL (0.5) is higher than male (0.4), So generally females still have more revisions than males but this difference is statistically insignificant in isolated cleft lip cases and this difference in number of lip and nose revisions become significant in cases with UCLP and BCLP .

This difference in secondary revisions in cases with isolated cleft lip [UCL mean 0.22 in males 0.33 in females, BCL mean 0.4 in males 0.5 in females] and those with cleft lip and palate [UCLP mean is 0.06 in males and 0.18 in females, BCLP mean is 0.16 in males and 0.43 in females], can be explained by the severity of cases, As cleft lip and palate cases need long time of surgical care and the number of operations needed for repair in cases of cleft lip and palate is high so the parents and patients tend to avoid more operations as aesthetical lip revisions in males than in females with over all lower means of secondary lip procedures in cleft lip and palate than isolated cleft lip, so we can say that there is insignificant difference in care of cleft lip cases whether unilateral or bilateral but with higher means of operation number than lip secondary care in UCLP and BCLP instead of the statistically significant difference. ( Table 1)

\section{Conclusion}

This gender based study found that females receive more surgical care than males do in all cleft varieties, but this increased number of surgical occasions is statistically significant only in secondary lip revisions in cases with unilateral or bilateral cleft lip and palate So revision of motivations for surgical corrections of post-repair deformities in both males and females should be done to insure the best care for cases without discrimination, also adding a pediatric psychiatrist to cleft multidisciplinary team is mandatory for better care of cases.

\section{References}

[1]. Dixon MJ, Marazita ML, Beaty TH, Murray JC. Cleft lip and palate: synthesizing genetic and environmental influences. Nat Rev Genet. 2011 Mar;12(3):167-78. PubMed PMID: 21331089.

[2]. Canfield MA, Honein MA, Yuskiv N, Xing J, Mai CT, Collins JS, et al.National estimates and race/ethnic-specific variation of selected birth defects in the United States, 1999-2001. Birth Defects Res A Clin Mol Teratol. 2006 Nov;76(11):747-56. PubMed PMID: 17051527.

[3]. Kirschner RE, LaRossa D. Cleft lip and palate. Otolaryngol Clin North Am. 2000 Dec;33(6):1191-215. PubMed PMID: 11449783.

[4]. Oakley A. Sex, gender and society. Ashgate Publishing, Ltd,UK.2015;182.

[5]. Johnson JL, Greaves L, Repta R. Better science with sex and gender: Facilitating the use of a sex and gender-based analysis in health research. Int J Equity Health. 2009 May 6;8:14. PubMed PMID: 19419579.

[6]. Vlassoff C. Gender differences in determinants and consequences of health and illness. J Health Popul Nutr. 2007 Mar;25(1):47-61. PubMed PMID: 17615903.

[7]. Regitz-Zagrosek V. Sex and gender differences in health. Science \& Society Series on Sex and Science. EMBO Rep. 2012 Jun 29;13(7):596-603. PubMed PMID: 22699937.

[8]. Vlassoff C, Garcia Moreno C. Placing gender at the centre of health pro- 
gramming: challenges and limitations. Soc Sci Med. 2002 Jun;54(11):171323. PubMed PMID: 12113453.

[9]. Popay J, Bartley M, Owen C. Gender inequalities in health: social position, affective disorders and minor physical morbidity. Soc Sci Med. 1993 Jan;36(1):21-32. PubMed PMID: 8424181

[10]. Mani M, Carlsson M, Marcusson A. Quality of life varies with gender and age among adults treated for unilateral cleft lip and palate. Cleft Palate Craniofac J. 2010 Sep;47(5):491-498. PubMed PMID: 20180705.

[11]. Kjellberg LS. A comparison of surgical treatment between male and female patients with complete unilateral cleft lip and palate in Västra Götaland region. 2013

[12]. Sinko K, Jagsch R, Prechtl V, Watzinger F, Hollmann K, Baumann A. Evaluation of esthetic, functional, and quality-of-life outcome in adult cleft lip and palate patients. Cleft Palate Craniofac J. 2005 Jul;42(4):355-61. PubMed PMID: 16001915.

[13]. Marcusson A, Paulin G, Ostrup L. Facial appearance in adults who had cleft lip and palate treated in childhood. Scand J Plast Reconstr Surg Hand Surg. 2002;36(1):16-23. PubMed PMID: 11925823. 